\title{
Philosophiques
}

\section{Maintenant la finitude. Une critique épistémologique du matérialisme spéculatif}

\section{Michel Bitbol}

Volume 47, numéro 2, automne 2020

URI : https://id.erudit.org/iderudit/1075131ar

DOI : https://doi.org/10.7202/1075131ar

Aller au sommaire du numéro

Éditeur(s)

Société de philosophie du Québec

ISSN

0316-2923 (imprimé)

1492-1391 (numérique)

Découvrir la revue

Citer ce document

Bitbol, M. (2020). Maintenant la finitude. Une critique épistémologique du matérialisme spéculatif. Philosophiques, 47(2), 417-426.

https://doi.org/10.7202/1075131ar d'utilisation que vous pouvez consulter en ligne.

https://apropos.erudit.org/fr/usagers/politique-dutilisation/ 


\title{
Disputatio
}

\section{Maintenant la finitude. Une critique épistémologique du matérialisme spéculatif}

\author{
MICHEL BITBOL \\ Archives Husserl, CNRS/ENS, 45 rue d'Ulm, Paris
}

Le «matérialisme spéculatif », variété française du réalisme spéculatif ${ }^{1}$ soutenue par Quentin Meillassoux, reste perçu comme l'une des rares nouveautés philosophiques de ce début du vingt et unième siècle. Il l'est sans doute par son ardeur iconoclaste, par le défi qu'il jette à trois cents ans d'histoire kantienne puis post- et néo-kantiennes de la philosophie, et par l'audace avec laquelle il prétend avoir accompli une percée métaphysique. Mais son défi est mal fondé, comme j'ai essayé de le montrer dans mon livre Maintenant la finitude.

La terre nouvelle que le matérialisme spéculatif espère avoir atteinte est celle d'une approche rationnelle de l'absolu. Après de longues ères de critique du rêve grec de l'épistémè, après que des générations de penseurs ont prescrit un geste réflexif pour identifier ce que le contenu de la connaissance doit aux moyens de connaître, l'affirmation que l'absolu est de quelque manière connaissable, et qu'une caractérisation précise peut en être établie, a quelque chose de bouleversant. La médiation des formes de la sensibilité, des catégories de l'entendement, de l'expérience vécue, des schèmes d'action, des formes symboliques, et tout particulièrement du langage, est déclarée surmontée. Un aperçu immédiat sur la chose que ces procédés dissimulaient en prétendant la révéler, semble alors à portée de la main.

L'émotion que suscite cette promesse est comparable à celle que Husserl a déclenchée au début du siècle précédent par son célèbre slogan du «retour aux choses mêmes». À ceci près que le mouvement préconisé est diamétralement opposé à celui qu'a inauguré la phénoménologie.

La phénoménologie neutralise la problématique dualiste de la chose en soi et des connaissances relatives à nous, l'opposition entre le texte du monde et sa grille de lecture catégoriale ou pragmatique. Elle désigne le phénomène lui-même comme ce que s'efforce d'élucider la connaissance, voire comme ce qui seul est. Pour marteler cette thèse en peu de mots, il

1. Comme mouvement de pensée, le réalisme spéculatif est né en avril 2007, lors d'un congrès à Londres qui réunissait quelques-uns de ses principaux créateurs: Quentin Meillassoux, Ray Brassier, Ian Hamilton Grant, et Graham Harman. Voir I. Thomas-Fogiel, «L'actuelle confrontation entre réalisme et idéalisme : la place de Fichte chez Brassier et Meillassoux ». Conférence prononcée dans le cadre du Workshop consacré à «la réception contemporaine de l'idéalisme allemand » à l'Université d'Ottawa, le 1 1er décembre 2017. 
suffit de citer Eugen Fink, selon qui «(la phénoménologie) affirme simplement que l'être est identique au phénomène ${ }^{2} »$. Si une trace des relativités épistémiques reste impliquée par la phénoménologie, elle se trouve résorbée dans la structure relationnelle de l'expérience, c'est-à-dire dans l'intentionnalité, qui est sa distension propre, son être-dirigé-vers-quelque-chose rendant inséparables la direction et la chose visée dans leur polarité même.

Le mouvement de la phénoménologie s'apparente ici à celui qu'Ernst Cassirer identifie dans la physique contemporaine: «À l'objection des sceptiques suivant laquelle nous ne pouvons jamais connaître les propriétés absolues des choses, la science répond ainsi: elle définit le concept de propriété de telle façon que ce dernier contienne en lui le concept de relation ${ }^{3}$.» Autrement dit, il n'est plus question de s'efforcer vers les choses telles qu'elles sont à travers la simple médiation des phénomènes qui surgissent de notre interaction avec elles, ce qui suscite un doute sceptique à propos de la fidélité de notre description de ces choses hypothétiques. Au lieu de cela, nous suspendons l'hypothèse de la chose, nous redirigeons notre effort de connaissance vers le fruit phénoménal de l'interaction présumée, et cela suffit à trancher le doute sceptique à la racine.

La dualité kantienne (et pré-kantienne) de la chose en soi et du phénomène est ainsi suspendue à l'occasion d'un geste de retour vers son origine et son en-deçà. Toute la problématique de la chose et de son phénomène, de la transcendance et de sa trace immanente s'en trouve renversée. Car ce sont ici certains traits internes du phénomène qui opèrent comme s'ils faisaient signe vers un extérieur de lui-même; c'est dans l'immanence que se constitue une imputation de transcendance.

À rebours de ce non-dualisme phénoménologique, le réalisme spéculatif prend à la lettre la dualité de la chose en soi et du phénomène, de l'être et de l'apparence, qui est posée par Kant dans la Dissertation de 1770 puis dans l'Esthétique transcendantale de la Critique de la raison pure. Il se démarque du Kant critique non pas tant parce qu'il pense pouvoir lire le phénomène comme le signe d'autre chose, que parce qu'il déclare pouvoir (à la manière du Kant pré-critique) transgresser le phénomène vers ce dont il est le signe par une modalité de la raison, et parce qu'il se proclame dès lors capable de dévoiler un trait fondamental de ce qu'il y a par-delà ce qui apparaît. Le matérialiste spéculatif redouble ce dualisme archétypal de l'être et de l'apparaître en adhérant à la dichotomie cartésienne entre qualités primaires et secondaires, rapportées respectivement aux structures mathématiques et aux qualités sensibles ${ }^{4}$.

Si l'imputation d'inconnaissabilité de la chose en soi est abolie, c'est donc ici par son au-delà et par son but ultime; ce n'est pas, comme en phé-

2. E. Fink, Proximité et distance, Jérôme Millon, 1994, p. 120.

3. E. Cassirer, La théorie de la relativité d'Einstein, Cerf, p. 68.

4. ALF, p. 16. 
noménologie, par son en-deçà et son origine. Si la croyance en l'inaccessibilité de la chose en soi est abolie, c'est en sautant par-dessus la problématique de l'accès et en s'installant d'emblée dans la chose par le biais des mathématiques tenues pour immédiatement descriptives de ses traits essentiels. Telle est la raison pour laquelle le réalisme spéculatif s'affirme un authentique réalisme, un réalisme ontologique, un "réalisme réel ${ }^{5}$ ": il prétend dévoiler quelque chose d'une réalité absolument indépendante de l'acte de dévoilement. Il assume ainsi le rêve originaire de la métaphysique, au lieu de s'en laisser dissuader par l'avertissement de Kant.

Mais comment le réaliste spéculatif fait-il pour ne pas s'échouer sur les écueils mis au jour par la critique kantienne? En déclarant tirer avantage de l'obstacle méthodologique opposé à l'entreprise métaphysique, au lieu de se laisser arrêter par lui.

Examinons ce point. Qu'est-ce que la métaphysique, et quelles sont les difficultés qui l'entravent? Selon Emmanuel Levinas: "Le désir métaphysique n'aspire pas au retour, car il est désir d'un pays où nous ne nacquîmes point. D'un pays étranger à toute nature, qui n'a jamais été notre patrie et où nous ne nous transporterons jamais ${ }^{6}$. " Le pays de la métaphysique n'est effectivement pas le nôtre. Il n'est pas le pays actuel de ce qui se montre à nous, mais un pays virtuel de ses hypothétiques coulisses. Il n'est pas le pays que nous habitons, mais un pays que nous pensons, un noumène. Or Kant n'a-t-il pas montré que cette prétention de percer vers un au-delà de l'apparaître outrepasse les pouvoirs de la faculté humaine de penser? La faculté humaine de penser n'a-t-elle pas selon lui pour unique fonction de lier les apparitions les unes aux autres par des lois universelles et nécessaires, qui sont du même coup les règles constitutives de ses objets? Et s'il en va ainsi, l'entreprise métaphysique n'est-elle pas purement spéculative; ne revientelle pas à construire des scénarios arbitraires (et parfois mutuellement contradictoires) de mondes possibles, plutôt qu'à $r e$-construire rigoureusement le scénario supposé unique du monde réel?

Telle est justement la prise inattendue dont se saisit le matérialiste spéculatif dans son ascension de la montagne «réalité ». Il pense en effet pouvoir sortir par le haut de l'impasse de la métaphysique, en identifiant le monde réel avec un ensemble non limitatif de possibles. Comme l'a écrit de façon concise un commentateur de Meillassoux, le matérialisme spéculatif ne s'occupe "pas tant des choses qui sont, que de la possibilité qu'elles puissent toujours être autrement ${ }^{7} »$. Il suffit de supposer que le monde réel n'est rien d'autre qu'un principe générateur illimité de tous les possibles, pour que la spéculation (sur les possibles) devienne un savoir (du monde

5. P. Kitcher, «Real Realism: the Galilean Strategy», The Philosophical Review, 110, 151-197, 2001.

6. E. Levinas, Totalité et infini, Livre de Poche, 2003, p. 22.

7. P. Hallward, «Tout est possible», Revue internationale des livres et des idées, 9, 209. 
réel). Encore faut-il avoir de bonnes raisons de faire à ce point crédit à la raison spéculative, et c'est donc sur ces méta-raisons que se concentre Meillassoux. On peut nommer de telles méta-raisons «l'argument-maître» du matérialisme spéculatif: il en sera question plus tard.

Avant cela, je voudrais souligner que l'option de Meillassoux, celle d'une permissivité sans limite à l'égard des possibles, est loin d'être le seul scénario de monde auquel aboutissent les réalismes spéculatifs. C’est dans la multiplicité de ces scénarios que se manifeste la part d'arbitraire de leurs reconstructions rationnelles.

La première option choisie par certains défenseurs du réalisme spéculatif consiste à adhérer sans discussion aux récits interprétatifs les plus courants des sciences objectives de la nature, et à les considérer comme ce qu'ils prétendent parfois être: une description asymptotiquement fidèle du monde tel qu'il est. Mais élever ainsi l'objectivité au rang de critère unique d'une ontologie est discutable, au regard de la dissociation kantienne entre objets et choses, entre objectivité (constituée) et réalité (donnée). De plus, la survalorisation ontologique de l'objectivité a des conséquences mal maîtrisées sur la place que nous assignons à notre humanité concrète dans le monde. Comme l'a écrit Erwin Schrödinger (après Kierkegaard et le courant existentialiste), «le tableau scientifique du monde ne contient par lui-même aucune valeur éthique, aucune valeur esthétique, aucun mot sur notre but ultime ou notre destin ${ }^{8} »$. Le tableau scientifique du monde est muet et vide. Il est vide de ce que les êtres connaissants ont dû mettre à l'écart pour constituer un domaine d'objectivité, à savoir eux-mêmes en tant que sujets d'expérience. Autrement dit, le tableau scientifique du monde est mort par construction; il est mort à la suite de l'extrusion du vécu (auquel un processus biologique ne saurait servir de substitut). Cette conséquence est ouvertement assumée par Ray Brassier, l'un des principaux auteurs du courant réaliste spéculatif. "Tout est déjà mort ${ }^{9}$ ", écrit-il, en assumant froidement le reproche que faisait Fichte aux réalismes métaphysiques de l'âge de la science: celui d'assimiler la réalité à l'être-objectif «fermé en soi et donc mort ${ }^{10}$ ». Selon Brassier, la nature des choses s'identifie à l'inertie de la mort, le vivant n'en représente que l'improbable et éphémère écart, et le désir de connaissance ne manifeste que le tropisme du vivant à l'égard du paysage mort dont il a un jour émergé.

La seconde option, choisie par d'autres défenseurs du réalisme spéculatif, diffère de la précédente du tout au tout. Elle consiste à infuser une intériorité dans les choses visibles, à la manière d'un envers du décor. Kant avait déjà esquissé cette piste vers le noumène; mais, contrairement à Leibniz, qui l'avait systématisée dans la Monadologie, il avait évité de la suivre par principe, exigeant que l'entendement s'en tienne à la «substance

8. E. Schrödinger, La nature et les Grecs, Les Belles Lettres, 2014.

9. R. Brassier, Nihil Unbound, Palgrave McMillan, 2007, p. 239.

10. J. G. Fichte, La théorie de la science, exposé de 1804, op. cit., p. 103. 
phénoménale». Kant remarquait en effet que, s'il fallait surmonter coûte que coûte la limitation de la connaissance aux seuls phénomènes, s'il fallait tâcher de remonter malgré elle vers les déterminations propres des choses et du monde, le seul modèle qui s'offrirait à nous serait celui de notre propre intériorité ${ }^{11}$. L'intérieur, notre intérieur, est l'unique étalon de l'intériorité des choses et du monde, car il en est l'unique lieu d'auto-donation. Graham Harman, cofondateur du courant réaliste spéculatif, prend acte de ce modèle de la nature propre des choses qu'est notre intériorité, et il l'étend, dans le sillage de Leibniz, à l'ensemble des objets. On a envie de dire qu'il retourne le slogan de Ray Brassier "tout est déjà mort " en son opposé, "tout est déjà vivant». C'est sur cette base néo-leibnizienne que Harman défend ce qu'il appelle une "ontologie orientée objet ${ }^{12}$ ».

Foisonnement de possibles, mort et désolation, ou fourmillement de vie, le moins qu'on puisse dire est que l'ontologie des réalismes spéculatifs n'est pas consensuelle. Leur unique point de convergence est l'assentiment qu'ils donnent à l'ontologie, et leur refus d'une philosophie de l'accès partiel, relatif, fini, ne laissant entrevoir qu'une ombre de ce qui est.

C'est pourquoi je m'en tiendrai ici, comme je l'ai fait dans mon livre, à leur version française, Le matérialisme spéculatif de Quentin Meillassoux. Pour exposer la contre-argumentation que j'ai élevée face à cette doctrine, je dresserai la liste de quatre de ses principaux arguments.

Le premier argument, le plus profond, mais aussi le plus difficile à maîtriser, est celui qu'on appellera l' "argument-maître ». Il consiste à vouloir prendre l'adversaire archétypal du matérialiste spéculatif en flagrant délit de contradiction performative. Cet adversaire archétypal est qualifié de "corrélationniste", pour dénoter sa propension supposée à affirmer que nous, étants finis, ne pouvons accéder à rien d'autre qu'au produit (phénoménal) de corrélations perspectivistes entre nous-mêmes et ce qu'il y a.

L'«argument-maître » est nommé ainsi par analogie et opposition au «maître-argument» de Berkeley en faveur de l'immatérialisme. Voici ce qu'écrit Berkeley: "(Cela) ne montre pas que vous puissiez concevoir la possibilité, pour les objets de votre pensée, d'exister hors de l'esprit. Pour l'établir, il serait nécessaire que vous les conceviez comme des existants non conçus et pensés, ce qui est une contradiction manifeste ${ }^{13}$. " L'analogie entre le maître argument de Berkeley et l'argument maître de Meillassoux vient de leur procédé. Ce procédé commun est avant tout dialogique et ad hominem.

11. I. Kant, Critique de la raison pure, B 321, Garnier-Flammarion, 2006. "Comme objet de l'entendement pur, toute substance doit au contraire posséder des déterminations et des facultés internes qui participent de sa réalité intérieure. Mais que puis-je penser, à titre d'accidents intérieurs, sinon ceux que me présente mon sens interne, c'est-à- dire ce qui est soimême une pensée, ou bien est analogue à cette pensée?"

12. G. Harman, Object-Oriented Ontology, Pelican, 2018.

13. G. Berkeley, Principles of Human Knowledge $\$ 23$, cité et traduit par G. Bryckman, Berkeley et le voile des mots, Vrin, 1993, p. 260. 
Il opère dans le dialogue avec un contradicteur, et il s'adresse explicitement à lui. Dans la phrase précédente, Berkeley commence par énoncer la thèse de son contradicteur: "la possibilité, pour les objets de votre pensée, d'exister hors de l'esprit». Puis il demande à ce contradicteur de s'avancer sur la scène du débat, d'oser affirmer la thèse précédente dans sa version la plus manifestement auto-contradictoire: penser un objet non pensé, concevoir un existant non conçu. Si ce contradicteur ne peut ni penser ni concevoir une telle chose, il a perdu car il a avoué que cela ne peut pas être. Et s'il déclare ouvertement pouvoir penser ou concevoir une telle chose, il a perdu aussi, car il tombe à l'instant dans l'absurdité. C'est ce piège dialectique tendu par Berkeley que Meillassoux appelle le «cercle corrélationnel». Il l'appelle ainsi parce qu'il s'agit d'une sorte de cercle vicieux, où celui qui veut sortir de sa propre corrélation avec ce qui est, afin d'en atteindre l'essence propre, doit se corréler avec cette hypothétique essence propre. Telle est la classe d'arguments, aussi simple qu'apparemment imparable, que Meillassoux veut désamorcer. Mais Meillassoux ne se contente pas de suspendre ou d'affaiblir ce «cercle corrélationnel »; il entreprend de le retourner comme un gant, et de tendre un contre-piège où est censé tomber son adversaire corrélationniste dès qu'il s'avance pour affirmer sa propre thèse. De même que le corrélationniste avait cru pouvoir défaire le réaliste au moyen d'une démonstration par l'absurde et d'une imputation de contradiction performative, le réaliste pense pouvoir défaire le corrélationniste par une contre-démonstration par l'absurde, et une contre-imputation de contradiction performative.

Peut-on réfuter cette réfutation du corrélationnisme, peut-on prouver que le matérialiste spéculatif n'est pas autorisé à bâtir un si haut édifice métaphysique à partir de l'aveu extorqué au corrélationniste? Deux grandes classes de réfutations de l'argument maitre du matérialiste spéculatif ont été proposées. L'une, due à Raphaël Millière ${ }^{14}$, est d'ordre logique et pragmatique, et l'autre, celle que j'ai proposée, est d'ordre pragmatique et existentiel. La réfutation logico-pragmatique joue sur l'usage des modalités: si l'on admet qu'une chose en soi non perçue est imaginable, voire possible, cela ne suffit pas à conclure qu'elle est réelle. La réfutation pragmatico-existentielle revient pour sa part à montrer que la contradiction performative du matérialiste est plus profonde, parce que plus immédiate (au sens d'immédiatement vécue), que celle du corrélationniste qui n'est que d'ordre discursif. Mais les deux réfutations soulignent cette évidence: aussi élégant que soit le mouvement tournant du matérialisme spéculatif pour penser un absolu, ce mouvement ne l'a pas fait sortir un instant de sa pensée. Son absolu n'est donc qu'un faux absolu, un «absolu relatif » à sa pensée, analogue au Dieu soi-disant «déduit» par la preuve ontologique.

14. R. Millière, «Réalisme et contradiction pragmatique. À propos des (re)lectures contemporaines du "maître argument" de Berkeley ", 2013, [en ligne: http://atmoc.free.fr/ resources/Milliere-Realisme-et-contradiction.pdf]. 
Le deuxième argument consiste en un retournement et une dévalorisation de la démarche critique de Kant. Auto-glorifiée comme une « révolution copernicienne » en philosophie, elle est requalifiée de «réaction ptolémaïque » (ou plutôt ptoléméenne) par Meillassoux. La philosophie critique de Kant est accusée par là d'avoir occasionné une régression philosophique qui fait bon marché de l'avancée philosophique implicite prêtée aux sciences de la nature. Mais l'erreur de Meillassoux est ici évidente: il a mal évalué le sens de la révolution de Copernic, qui insiste davantage sur la relativisation du mouvement apparent des astres à l'observateur terrestre, que sur la perte de centralité de la demeure de l'être humain. Et il ne voit pas, à partir de là, à quel point la relativisation kantienne des déterminations de l'objet de science est fidèle à l'esprit du copernicanisme.

Le troisième argument fait justement fond sur la dévalorisation des sciences modernes de la nature qu'est supposée impliquer leur lecture phénoménologique ou critique, et lui oppose leur hyper-valorisation réaliste. Meillassoux commence prudemment par demander d'accorder au chercheur scientifique que ses énoncés puissent être littéralement vrais. Ce à quoi il tient est qu'on évite de dénigrer d'emblée la prétention à la vérité absolue des énoncés scientifiques, qu'on évite de déclarer qu'ils ne sauraient valoir que relativement à une opération expérimentale, à un système conceptuel, à un schème cognitif, ou à un paradigme théorique. Le fait de leur attribuer un statut seulement hypothétique ne déroge pas à cette prescription, puisqu'une hypothèse est une thèse avant la thèse, une thèse qui s'expose certes à la réfutation, mais aussi, potentiellement, à la vérification. Derrière cet écran de prudence prennent forme des affirmations bien plus fortes sur la vérité des propositions scientifiques, dont presque toutes portent la marque d'un platonisme mathématique. Il suffit de lire ces quelques propositions extraites de divers chapitres d'Après la finitude: "ce qui est mathématisable est absolutisable ${ }^{15}$ »; les «conditions non quelconques» transcrites dans les mathématiques peuvent représenter " autant de propriétés absolues de ce qui est » $^{16}$; "tout ce qui, de l'objet, peut être formulé en termes mathématiques, il y a sens à le penser comme propriété de l'objet en $\operatorname{soi}^{17}{ }^{1}$. Mais hors du platonisme, dans le cadre de conceptions alternatives des mathématiques, comme l'idéalisme transcendantal, le pragmatisme, l'intuitionnisme ou le constructivisme, les propositions citées perdent tout caractère d'évidence. Les mathématiques se contentent alors de recueillir les conditions de possibilité d'une connaissance intersubjectivement ou interpositionnellement valide des phénomènes, ou bien d'exprimer l'ordre des actions humaines possibles et d'encadrer ainsi toute action humaine effective.

15. ALF, p. 175.

16. ALF, p. 90.

17. ALF, p. 16. 
Le principal témoin convoqué à l'appui de la légitime prétention à la vérité des énoncés scientifiques est « l'énoncé ancestral », c'est-à-dire l'énoncé sur des événements passés ayant précédé l'existence de l'espèce humaine. L'argument de Meillassoux sur les énoncés ancestraux s'appuie sur deux étapes d'appel au bon sens.

D'une part, lorsqu'un chercheur scientifique affirme que le Big Bang de l'univers a eu lieu il y a environ 13,5 milliards d'années, ou que les dinosaures se sont éteints il y a 65 millions d'années, il sous-entend que ces énoncés sont ou pourraient être littéralement vrais; que ces événements-là sont réellement advenus à un certain moment et en un certain lieu de l'espace-temps réel. Lui refuser cette prétention à la vérité littérale de ses énoncés, déclarer que de tels énoncés sont seulement des corrélats de ses questions, de ses catégories, ou de ses procédés expérimentaux, ce serait les priver de sens, et surtout le priver du sens qu'il souhaite leur donner. Le chercheur scientifique soumis à un tel refus aurait l'impression de ressembler à Roquentin, l'historien dont Sartre a fait le héros de son roman La nausée. Pour lui, comme pour l'historien fictif, la phrase énonçant l'advenue d'un événement passé ne serait rien de plus qu'une suite de traces écrites résumant platement d'autres traces, archéologiques, instrumentales, ou archivées.

D'autre part, lorsqu'un chercheur scientifique affirme que le Big Bang de l'univers a eu lieu il y a environ 13,5 milliards d'années, il n'est pas question pour lui d'atténuer son affirmation en précisant qu'elle est relative au genre humain qui, par son travail, a été capable d'assembler les moyens de la formuler et de l'attester. Car le genre humain était évidemment absent de l'univers à sa naissance. L'affirmation du chercheur scientifique a donc nécessairement, selon le matérialiste spéculatif, une prétention à la validité absolue.

Je me suis attardé un peu plus longtemps sur cet argument, parce qu'en lui se manifeste ce qui me semble être le principal ressort du geste philosophique de Meillassoux: le désir de refonder une philosophie qui soit parfaitement en phase avec l'époque des sciences; et la volonté associée de rejeter des philosophies, comme celle de Husserl dans la Crise des sciences européennes, ou de Michel Henry dans La barbarie, qui ont la réputation d'être anti-scientifiques.

L'une des erreurs les plus évidentes que je prête ici au matérialisme spéculatif est de croire qu'une philosophie de l'âge des sciences est forcément une philosophie prenant au pied de la lettre les propositions exotériques des scientifiques, celles qu'ils prononcent au terme de leur recherche ou à destination du grand public. Or, comme le disait Einstein, si l'on veut comprendre la signification de la quête scientifique, il faut "prendre en considération ce que les scientifiques font, et non pas ce qu'ils disent à propos de ce qu'ils font ${ }^{18}{ }^{1}$. Le discours exotérique des chercheurs n'est

18. «A. Einstein, Herbert Spencer lecture», Oxford, 10 June 1933, cité dans T. Ryckman, The Reign of Relativity, Oxford University Press, 2005, p. 81. 
qu'un petit extrait symbolique de leurs travaux, reformaté selon la grammaire du langage courant et selon une métaphysique héritée bon gré mal gré des gestes platonico-aristotéliciens. Leur discours public n'est que la partie émergée, et déformée, de l'iceberg de la compréhension qu'ils ont acquise de la part manipulable de notre environnement. Pour aller au-delà de cette vue superficielle de la science, il faut plonger dans l'activité théoréticoexpérimentale des chercheurs, voir ce qu'ils font de leurs symbolismes mathématiques et comment ils les articulent à leur usage des instrumentations. Or, comme j'ai entrepris de le montrer au chapitre VII de Maintenant la finitude, la considération détaillée de cette activité théorético-expérimentale en physique quantique est fatale à la thèse du matérialiste spéculatif.

Le quatrième argument du matérialisme spéculatif, enfin, est d'ordre théologico-politique. En imposant des bornes au domaine rationnel, en excluant toute pensée de l'absolu, le corrélationnisme est accusé de laisser le champ libre à un discours irrationnel sur l'absolu. Il faut admettre que certaines formulations de Kant semblent donner prise à cette critique. N'a-t-il pas écrit, pour montrer comment il est parvenu à articuler la raison pure et la raison pratique: "je devais donc supprimer le savoir afin de trouver une place pour la $f_{0} i^{19}$ " ? Selon Kant, c'est en comprenant que le savoir scientifique n'atteint pas la chose en soi et se borne à de "simples phénomènes ${ }^{20}$ ", qu'on ménage un espace aux postulats de la raison pratique, et qu'on permet à la foi de leur offrir l'armature de ses contenus symboliques. L'énigme résiduelle de la chose en soi crée un vide qui appelle un remplissement "fidéiste ", et c'est cette lacune que vise donc à combler le matérialisme spéculatif.

Mais, en niant toute possibilité de se saisir de l'absolu comme objet de pensée, le corrélationnisme ne laisse-t-il vraiment d'autre option que l'irrationalité de la foi ? Pas forcément. Plusieurs variétés de corrélationnismes réservent une place insigne à l'absolu. Mais une place localisable nulle part, parce qu'elle est partout ${ }^{21}$, et un statut qui n'est celui d'aucun objet existant ou inexistant, accessible ou inaccessible pour la pensée, en-deçà ou au-delà de la limite du pensable, corrélé à la pensée ou identifié à la corrélation. L'absolu n'est, pour ces corrélationnistes, ni (bien entendu) le thème d'une pensée effective, ni le thème d'une pensée qui le situerait hors de son champ de validité. Leur absolu excède et transit la pensée parce que la pensée en participe; il n'a rien d'un objet parce qu'objectiver en relève; il n'est pas un étant parce qu'être revient à en être. L'absolu du corrélationniste n'est pas un objet pensable parce qu'il pré-conditionne la pensée.

19. E. Kant, Critique de la raison pure, B XXX, in E. Kant, CEuvres philosophiques I, op. cit. p. 748.

20. Ibid. B XXIX, p. 747.

21. L'allusion à la caractérisation de Dieu par Blaise Pascal, dans ses Pensées, n'est pas fortuite: "C'est une sphère infinie dont le centre est partout, la circonférence nulle part » (Édition Brunschwig, II, 72-73). 
Mon livre Maintenant la finitude vise non seulement à réfuter les arguments précédents un par un, mais aussi à identifier la source commune de leur faiblesse. Cette source commune, comme cela transparaît des réflexions précédentes, est un défaut de réflexivité. Le matérialiste spéculatif échoue à prendre conscience des conditions d'assertabilité de ses arguments, c'est-àdire de la situation du sujet vivant, parlant, connaissant, et capable de se « relier» qu'il est lui-même... apparemment à son insu. 